UCB-PTH-05/27

LBNL-58857

\title{
Minimally Fine-Tuned Supersymmetric Standard Models with Intermediate-Scale Supersymmetry Breaking
}

\author{
Yasunori Nomura, David Poland, and Brock Tweedie \\ Department of Physics, University of California, Berkeley, CA 94720 \\ Theoretical Physics Group, Lawrence Berkeley National Laboratory, Berkeley, CA 94720
}

\begin{abstract}
We construct realistic supersymmetric theories in which the correct scale for electroweak symmetry breaking is obtained without significant fine-tuning. We consider two classes of models. In one class supersymmetry breaking is transmitted to the supersymmetric standard model sector through Dirac gaugino mass terms generated by a $D$-term vacuum expectation value of a $U(1)$ gauge field. In the other class the supersymmetry breaking sector is separated from the supersymmetric standard model sector in an extra dimension, and the transmission of supersymmetry breaking occurs through gauge mediation. In both these theories the Higgs sector contains two Higgs doublets and a singlet, but unlike the case for the next-to-minimal supersymmetric standard model the singlet field is not responsible for generating the supersymmetric or supersymmetry breaking mass for the Higgs doublets. These masses, as well as the mass for the singlet, are generated through gravitational-strength interactions. The scale at which the squark and slepton masses are generated is of order $(1 \sim 100) \mathrm{TeV}$, and the generated masses do not respect the unified mass relations. We find that electroweak symmetry breaking in these theories is caused by an interplay between the top-stop radiative correction and the holomorphic supersymmetry breaking mass for the Higgs doublets and that the fine-tuning can be reduced to the level of $20 \%$. The theories have rich phenomenology, including a variety of possibilities for the lightest supersymmetric particle.
\end{abstract}




\section{Introduction}

Weak scale supersymmetry is an attractive candidate for physics beyond the standard model. It not only stabilizes the Higgs potential against potentially large radiative corrections, but also leads to an elegant picture of gauge coupling unification at a scale $M_{X} \simeq 10^{16} \mathrm{GeV}$ [1]. Nondiscovery of the superparticles or the Higgs boson at LEP II, however, puts strong constraints on how supersymmetry can be realized at the weak scale. In the minimal supersymmetric standard model (MSSM), evading the lower bound on the physical Higgs boson mass typically requires a large top squark mass. This in turn gives a large radiative correction to the Higgs boson mass-squared parameter, leading to fine-tuning in electroweak symmetry breaking. In fact, the problem of fine-tuning is somewhat generic in theories with weak scale supersymmetry, and is called the supersymmetric fine-tuning problem.

Recently, a general framework has been discussed for supersymmetric theories that avoid fine-tuning while preserving the successful features of supersymmetry $[2,3]$. The key point is to lower both the top squark masses and the mediation scale of supersymmetry breaking, by violating simple unified mass relations. ${ }^{1}$ This makes radiative corrections to the Higgs masssquared parameter small, and thus reduces fine-tuning. A simple way to accommodate such light top squarks is to introduce an additional contribution to the Higgs quartic couplings other than that from the $S U(2)_{L} \times U(1)_{Y} D$-terms of the MSSM (for theories giving such a contribution, see e.g. $[4-11]){ }^{2}$ In Refs. [2, 3] these were achieved by adopting the mechanism of [14]: the dynamical supersymmetry breaking sector has a global $S U(5)$ symmetry, of which the $S U(3) \times$ $S U(2) \times U(1)$ subgroup is explicitly gauged and identified as the standard model gauge group, but this $S U(5)$ is spontaneously broken to the gauged subgroup at the dynamical scale of $\Lambda \approx(10 \sim$ 100) $\mathrm{TeV}$. This structure allows us to accommodate the successful prediction associated with gauge coupling unification at the leading-log level, while the unwanted unified mass relations among the squark and slepton masses are avoided. The mediation scale of supersymmetry breaking is very low and of order $\Lambda$, which is the scale of dynamical supersymmetry breaking itself. The Higgs sector superpotential is generated from interactions between the Higgs and dynamical supersymmetry breaking sectors through marginal operators.

In this paper we construct classes of explicit supersymmetric standard models in which the fundamental scale of supersymmetry breaking is an intermediate scale, $M_{I}=\sqrt{m_{\text {weak }} M_{\mathrm{Pl}}}$, and

\footnotetext{
${ }^{1}$ The unified mass relations need not be violated if the top quark and Higgs boson are both rather heavy, $m_{t} \simeq(180 \sim 182) \mathrm{GeV}$ and $M_{\text {Higgs }} \simeq(200 \sim 250) \mathrm{GeV}$ [3]. We do not consider this scenario in this paper.

${ }^{2} \mathrm{~A}$ solution to the supersymmetric fine-tuning problem that does not require an extension of the Higgs sector at the weak scale has recently been presented in [12], where the supersymmetry breaking mass for the up-type Higgs boson is suppressed by a cancellation between two different contributions [13], and a large stop mixing parameter and a small holomorphic supersymmetry breaking Higgs mass ensure successful electroweak symmetry breaking with relatively small top squark masses.
} 
yet the supersymmetric fine-tuning problem is ameliorated. An advantage of intermediate-scale supersymmetry breaking is that the Higgs sector superpotential is obtained relatively easily through nonrenormalizable interactions suppressed by the Planck scale [15]. To implement this mechanism without introducing the supersymmetric flavor problem, we consider two classes of theories. In one class supersymmetry breaking is transmitted to the supersymmetric standard model sector through a $D$-term vacuum expectation value (VEV) of a $U(1)$ gauge field [16]. In the other class the supersymmetry breaking sector is separated from the supersymmetric standard model sector in an extra dimension, and the transmission of supersymmetry breaking occurs through gauge mediation [17]. In both these theories the Higgs sector contains two Higgs doublets and a singlet. Our Higgs sector superpotential, however, differs from that of the nextto-minimal supersymmetric standard model: it contains weak-scale mass parameters which are naturally generated through gravitational-strength interactions. The scale at which the squark and slepton masses are generated is of order $(1 \sim 100) \mathrm{TeV}$, and the generated masses do not respect the unified mass relations. These features nicely meet the general criteria discussed above. (Note that the relevant scale for the fine-tuning argument is the scale at which the squark and slepton masses are generated, and not the one at which the gaugino masses are generated.)

Electroweak symmetry breaking in our theories occurs because of an interplay between the top-stop radiative correction and the holomorphic supersymmetry breaking mass squared for the Higgs doublets, the $\mu B$ term. The dynamics of the singlet field $S$ almost decouples from the electroweak symmetry breaking physics due to a relatively large supersymmetric mass for $S$. These theories, therefore, naturally realize the scenario II of $\mu B$-driven electroweak symmetry breaking discussed in Ref. [18]. This has an advantage, compared with theories based on the next-to-minimal supersymmetric standard model (NMSSM), that there is no strict requirement on the potential that the correct supersymmetric Higgs mass term ( $\mu$ term) should be reproduced by the VEV of the singlet field, thus opening up a larger region of parameter space that correctly breaks the electroweak symmetry. The $\mu$ and $\mu B$ terms of order the weak scale are naturally generated in our theories through gravitational-strength interactions. (For attempts of reducing fine-tuning in the context of the NMSSM, see e.g. [19].) In the present scheme, the amount of fine-tuning is essentially determined by the ratio of the lightest neutral Higgs-boson and the charged Higgs-boson squared masses. We find that the fine-tuning in these theories can be reduced to the level of $20 \%$.

The organization of the paper is as follows. In section 2 we discuss the first class of theories. We study electroweak symmetry breaking and the superparticle spectrum, identifying some characteristic features of the theories. In section 3 we discuss the second class of theories and perform a similar analysis of electroweak symmetry breaking and the superparticle spectrum. 
Conclusions are given in section 4 .

\section{Models with $D$-type Supersymmetry Breaking}

In this section we present the first class of models, in which supersymmetry breaking is transmitted to the supersymmetric standard model sector through a $D$-term VEV of a $U(1)$ gauge field. We find that the fine-tuning is reduced to the level of $20 \%$.

\subsection{Supersymmetry breaking from a $D$-term VEV}

The supersymmetric standard model sector of our theories contains, as usual, the $S U(3)_{C} \times$ $S U(2)_{L} \times U(1)_{Y}(321)$ gauge multiplet, $V_{i}\left(i=1,2,3\right.$ for $U(1)_{Y}, S U(2)_{L}$ and $\left.S U(3)_{C}\right)$, and three generations of matter fields, $Q, U, D, L$ and $E$. We also introduce a gauge singlet chiral superfield $S$ as well as two Higgs doublets $H_{u}$ and $H_{d}$, with the standard Yukawa couplings in the superpotential $W=y_{u} Q U H_{u}+y_{d} Q D H_{d}+y_{e} L E H_{d}$.

Following Ref. [16], we consider that supersymmetry breaking is transmitted to the supersymmetric standard model sector through a $D$-term VEV of a $U(1)$ gauge interaction, $U(1)^{\prime}$ :

$$
\left\langle V^{\prime}\right\rangle=\frac{1}{2} \theta^{2} \bar{\theta}^{2} D^{\prime}
$$

where $V^{\prime}$ is the vector superfield for $U(1)^{\prime}$. Introducing chiral superfields that transform as adjoints under $321, A_{1}(\mathbf{1}, \mathbf{1})_{0}, A_{2}(\mathbf{1}, \mathbf{3})_{0}$ and $A_{3}(\mathbf{8}, \mathbf{1})_{0}$, supersymmetry breaking in Eq. (1) is transmitted to the supersymmetric standard model sector through the following operators:

$$
\mathcal{L}=\sum_{i=1,2,3} \int d^{2} \theta \frac{\zeta_{i}}{M_{*}} \mathcal{W}_{i}^{\alpha} \mathcal{W}_{\alpha}^{\prime} A_{i}+\text { h.c. }
$$

where $\zeta_{i}$ are coefficients of $O(1), M_{*}$ is a mass parameter of order the Planck scale, and $\mathcal{W}_{i}^{\alpha}$ and $\mathcal{W}^{\prime \alpha}$ are the field-strength superfields for 321 and $U(1)^{\prime}$, respectively. ${ }^{3}$ These operators generate Dirac masses for the gauginos of order $D^{\prime} / M_{*}$, which in turn generate flavor-universal squark and slepton squared masses of order $\left(1 / 16 \pi^{2}\right)\left(D^{\prime} / M_{*}\right)^{2}$ at one loop. With $M_{*}$ of order the Planck scale, $D^{\prime} \sim\left(10^{10}-10^{11} \mathrm{GeV}\right)^{2}$. An important property of this transmission is that the squark and slepton masses are generated at the scale of Dirac gaugino masses $\approx D^{\prime} / M_{*} \sim(1-10) \mathrm{TeV}$, although the gaugino masses are present at the scale $M_{*}$. This reduces the logarithm associated

\footnotetext{
${ }^{3}$ We assume that $D^{\prime}$ is much larger than the largest $F$-type VEV, $F$, in the theory, i.e. $D^{\prime} \gg F$, so that the contributions to the supersymmetry breaking masses from $F$ are negligible. For a discussion on how to obtain $D^{\prime} \gg F$, see e.g. [20]. Alternatively, one can separate the field giving the largest $F$ from the supersymmetric standard model sector in an extra dimension; see e.g. [21]. (Mediation of supersymmetry breaking by Eq. (2) was also considered in $[22,3]$ in a slightly different context, in which the $A_{i}$ fields arise as composites.)
} 
with the top-Yukawa induced radiative correction to the Higgs soft supersymmetry breaking mass, and thus helps the reduction of fine-tuning.

We take the coefficients $\zeta_{i}$ in Eq. (2) to be free parameters. In particular, we do not impose any unified relations on the three coefficients $\zeta_{1}, \zeta_{2}$ and $\zeta_{3}$. This is necessary to break unwanted

unified mass relations for the squarks and sleptons, such as $m_{\tilde{t}}^{2} / m_{\tilde{e}}^{2} \approx\left(4 g_{3}^{4} / 3\right) /\left(3 g_{1}^{4} / 5\right)$, and to reduce fine-tuning. (For $\zeta_{1}=\zeta_{2}=\zeta_{3}$ in the basis where the gauge kinetic terms are given by $\mathcal{L}=\sum_{i} \int d^{2} \theta\left(1 / 4 g_{i}^{2}\right) \mathcal{W}_{i}^{\alpha} \mathcal{W}_{i \alpha}+$ h.c., which is expected to be the case in naive unified theories, the squarks and sleptons obey unwanted unified mass relations $m_{\tilde{f}}^{2} \propto \sum_{i} g_{i}^{4} C_{i}^{\tilde{f}}$, where $\tilde{f}=\tilde{q}, \tilde{u}, \tilde{d}, \tilde{l}, \tilde{e}$ and $C_{i}^{\tilde{f}}$ are the group theoretical factors.)

The introduction of the $A_{i}$ fields destroys the successful supersymmetric prediction for gauge coupling unification. Unification of the couplings can be recovered by the introduction of arbitrary vector-like matter fields, but at the price of losing the predictivity for the low-energy gauge couplings. A possibility of recovering the prediction is to use the trinification idea, which has been discussed in [16]. (The $S U(5)$ case leads to a Landau pole for the gauge couplings much below the unification scale at two loops.) This issue does not occur in the model presented in section 3, and we do not discuss it further in the context of the present model.

\subsection{Masses for the $A$ fields}

For $D^{\prime} \sim\left(10^{10}-10^{11} \mathrm{GeV}\right)^{2}$, the gravitino mass is roughly of the order of the weak scale: $m_{3 / 2} \sim D^{\prime} / M_{\mathrm{Pl}}$, where $M_{\mathrm{Pl}}$ is the reduced Planck scale. The precise value of $m_{3 / 2}$ depends on various unknown parameters, for example on $M_{*} / M_{\mathrm{Pl}}$, so here we take $m_{3 / 2}$ to be a free parameter of order $m_{3 / 2} \sim(100 \mathrm{GeV}-1 \mathrm{TeV})$. With these values of $m_{3 / 2}$, supersymmetric masses of order the weak scale can be naturally generated through the Kähler potential. For example, if the Kähler potential contains the term $K=\sum_{i} \lambda_{A_{i}} A_{i}^{2} / 2+$ h.c. $(i=1,2,3)$, where $\lambda_{A_{i}}$ are dimensionless coefficients, the supergravity Lagrangian produces the effective superpotential term

$$
W_{\text {eff }}=\frac{1}{2} \sum_{i=1,2,3} m_{A, i} A_{i}^{2},
$$

where $m_{A, i}=\lambda_{A_{i}} m_{3 / 2}$ [15]. This can be understood easily in the compensator formalism (see e.g. [23]), in which the above Kähler potential term can be written as

$$
\mathcal{L}=\int d^{4} \theta \frac{\phi^{\dagger}}{\phi} \sum_{i=1,2,3} \frac{\lambda_{A_{i}}}{2} A_{i}^{2}+\text { h.c. }
$$

in the normalization where $A_{i}$ are canonically normalized. Here, $\phi$ is the compensator field, which takes the value $\phi=1+\theta^{2} m_{3 / 2}$. We then find that Eq. (4) gives the supersymmetric mass term of Eq. (3) as well as the soft supersymmetry breaking term $\mathcal{L}_{\text {soft }}=-\sum_{i} m_{A, i} m_{3 / 2} a_{i}^{2} / 2+$ h.c., where 
$a_{i}$ are the lowest components of $A_{i}$. Note that, assuming real couplings, we still have a freedom of choosing the signs of $m_{A, i}$ (in the phase convention that $\zeta_{i}$ and $m_{3 / 2}$ are real and positive). Soft supersymmetry breaking terms could also receive contributions from the operators

$$
\mathcal{L}=\sum_{i=1,2,3} \int d^{2} \theta \frac{\eta_{i}}{2 M_{*}^{2}} \mathcal{W}^{\prime \alpha} \mathcal{W}_{\alpha}^{\prime} A_{i}^{2}+\text { h.c. }
$$

Together with the contributions from the Kähler potential of Eq. (4), we obtain

$$
\begin{aligned}
\mathcal{L}_{\text {soft }} & =-\frac{1}{2} \sum_{i=1,2,3}\left(m_{A, i} m_{3 / 2}-\frac{\eta_{i} D^{\prime 2}}{M_{*}^{2}}\right) a_{i}^{2}+\text { h.c. } \\
& \equiv-\frac{1}{2} \sum_{i=1,2,3} b_{A, i} a_{i}^{2}+\text { h.c.. }
\end{aligned}
$$

Here, as in the case of $\zeta_{i}$, we do not impose any unified relations on $\lambda_{A_{i}}$ or $\eta_{i}$. The conditions for quadratic stability of the $a_{i}$-field origin are given by

$$
\left|m_{A, i}\right|^{4}+4 g_{i}^{2}\left|m_{D, i}\right|^{2}\left|m_{A, i}\right|^{2}-\left|b_{A, i}\right|^{2}+2 g_{i}^{2}\left(b_{A, i} m_{D, i}^{* 2}+b_{A, i}^{*} m_{D, i}^{2}\right)>0
$$

where $m_{D, i} \equiv-i \zeta_{i} D^{\prime} / \sqrt{2} M_{*}$. We assume that Eq. (7) are satisfied for all $i=1,2,3$.

The introduction of a gauge singlet $A_{1}$ has a potential danger of destabilizing the gauge hierarchy. Specifically, the operator $\mathcal{L} \approx \int d^{4} \theta M_{*} \phi^{\dagger} A_{1}$, together with Eq. (3), could lead to a large VEV for $A_{1}$, and thus to a large Fayet-Iliopoulos $D$ term for $U(1)_{Y}$ through Eq. (2). ${ }^{4}$ Even if absent at tree level, this operator would be generated at radiative level under the presence of general nonrenormalizable operators. We avoid this by imposing a symmetry

$$
V^{\prime} \leftrightarrow-V^{\prime}, \quad A_{i} \leftrightarrow-A_{i}
$$

on the interactions of the observable sector. This symmetry is broken by the $D^{\prime}$ and physics generating it. However, if the breaking appears sufficiently soft in the observable sector, the dangerous operator linear in $A_{1}$ is sufficiently suppressed. Such a setup can naturally arise, for example, by generating $D^{\prime}$ on the infrared brane in warped space (with the infrared-brane scale set to $\approx D^{\prime}$ ) and transmitting it to the observable sector on the ultraviolet brane through a bulk $U(1)^{\prime}$. In the following, we assume that the operator linear in $A_{1}$ is sufficiently suppressed.

\subsection{The Higgs sector}

The Higgs sector of our theory consists of three chiral superfields $S(\mathbf{1}, \mathbf{1})_{0}, H_{u}(\mathbf{1}, \mathbf{2})_{1 / 2}$ and $H_{d}(\mathbf{1}, \mathbf{2})_{-1 / 2}$. There are some variations on possible interactions in the Higgs sector. Here, to demonstrate our point, we adopt a particular setup that uses a discrete $Z_{4, R}$ symmetry to constrain the form of these interactions. 


\begin{tabular}{|c|cc|ccccc|ccc|c|}
\hline & $V_{i}$ & $A_{i}$ & $Q$ & $U$ & $D$ & $L$ & $E$ & $H_{u}$ & $H_{d}$ & $S$ & $V^{\prime}$ \\
\hline$Z_{4, R}$ & 0 & 0 & 1 & 1 & 1 & 1 & 1 & 0 & 0 & 2 & 0 \\
\hline
\end{tabular}

Table 1: $Z_{4, R}$ charges for the fields.

We consider a discrete $R$ symmetry, $Z_{4, R}$, under which fields transform as in Table 1 . This charge assignment allows all the interactions discussed so far, including the Yukawa couplings and Eq. (2). ${ }^{5}$ In the absence of supersymmetry breaking, the Higgs sector superpotential consistent with $Z_{4, R}$ is $W_{0}=\lambda S H_{u} H_{d}+(\kappa / 3) S^{3}$. (We assume that the possible term linear in $S$ is absent.) In addition, we have terms arising from the Kähler potential $K=\lambda_{H} H_{u} H_{d}+\left(\lambda_{S} / 2\right) S^{2}+$ h.c.. Adding these together, the superpotential of our Higgs sector is given by

$$
W_{H}=\lambda S H_{u} H_{d}+\mu H_{u} H_{d}+\frac{M_{S}}{2} S^{2}+\frac{\kappa}{3} S^{3},
$$

where $\mu=\lambda_{H} m_{3 / 2}$ and $M_{S}=\lambda_{S} m_{3 / 2}$ are mass parameters of order the weak scale. ${ }^{6}$ Soft supersymmetry breaking parameters arise from the Kähler potential terms as well as from the operators $\mathcal{L}=\int d^{2} \theta\left(\eta_{H} H_{u} H_{d}+\eta_{S} S^{2} / 2\right) \mathcal{W}^{\prime \alpha} \mathcal{W}_{\alpha}^{\prime} / M_{*}^{2}+$ h.c., giving

$$
\begin{aligned}
\mathcal{L}_{H, \mathrm{soft}} & =-\left(\mu m_{3 / 2}-\frac{\eta_{H} D^{\prime 2}}{M_{*}^{2}}\right) H_{u} H_{d}-\frac{1}{2}\left(M_{S} m_{3 / 2}-\frac{\eta_{S} D^{\prime 2}}{M_{*}^{2}}\right) S^{2}+\text { h.c. } \\
& \equiv-b_{H} H_{u} H_{d}-\frac{b_{S}}{2} S^{2}+\text { h.c. }
\end{aligned}
$$

where we have used the same symbol for a chiral superfield and its scalar component for $H_{u}$, $H_{d}$ and $S$. The Higgs doublets also obtain non-holomorphic supersymmetry breaking masses at one loop through 321 gauge interactions.

\subsection{Parameters at the weak scale}

Contributions to the gaugino masses arise from the operators in Eqs. $(2,3)$. The masses of adjoint scalars also come from Eq. (6). Defining component fields as $V=-\theta^{\alpha} \sigma_{\alpha \dot{\alpha}}^{\mu} \bar{\theta}^{\dot{\alpha}} A_{\mu}-$ $i \bar{\theta}^{2} \theta^{\alpha} \lambda_{\alpha}+i \theta^{2} \bar{\theta}_{\dot{\alpha}} \lambda^{\dagger \dot{\alpha}}+(1 / 2) \theta^{2} \bar{\theta}^{2} D$ and $A=a+\sqrt{2} \theta^{\alpha} \psi_{\alpha}+\theta^{2} F$, these operators give

$$
\mathcal{L}=-m_{D} \lambda^{\alpha} \psi_{\alpha}-m_{D}^{*} \lambda_{\dot{\alpha}}^{\dagger} \psi^{\dagger \dot{\alpha}}+\sqrt{2} i m_{D} D a-\sqrt{2} i m_{D}^{*} D a^{\dagger}
$$

\footnotetext{
${ }^{4}$ The direct kinetic mixing between $U(1)^{\prime}$ and $U(1)_{Y}, \mathcal{L}=\int d^{2} \theta \mathcal{W}_{1}^{\alpha} \mathcal{W}_{\alpha}^{\prime}$, is assumed to be absent throughout.

${ }^{5}$ This $Z_{4, R}$ symmetry forbids dangerous dimension four and five proton decay operators, as well as a large tree-level supersymmetric mass for the Higgs doublets. It is broken by the VEV of the compensator field (a constant term in the superpotential needed to cancel the cosmological constant) to the $Z_{2, R}$ subgroup, which is nothing but the standard $R$ parity.

${ }^{6}$ The superpotential of Eq. (9) can also be written in the form of $W_{H}=\lambda S H_{u} H_{d}+f(S)$, where $f(S)$ is a general cubic function of $S$, by shifting the $S$ field as $S \rightarrow S-\mu / \lambda$.
} 


$$
\begin{aligned}
& -\frac{1}{2} m_{A} \psi^{\alpha} \psi_{\alpha}-\frac{1}{2} m_{A}^{*} \psi_{\dot{\alpha}}^{\dagger} \psi^{\dagger \dot{\alpha}}+m_{A} a F+m_{A}^{*} a^{\dagger} F^{\dagger} \\
& -\frac{1}{2} b_{A} a^{2}-\frac{1}{2} b_{A}^{*} a^{\dagger 2}-m_{a}^{2} a^{\dagger} a,
\end{aligned}
$$

for each gauge group factor $S U(3)_{C}, S U(2)_{L}$ and $U(1)_{Y}$ (we have suppressed the index $i=1,2,3$ ). Here, we have added non-holomorphic supersymmetry breaking masses for $a$ 's (the last term), although they are small in the present theory. The mass parameters $m_{D} \equiv-\left(i \zeta_{i} / \sqrt{2}\right)\left\langle D^{\prime}\right\rangle / M_{*}$, $m_{A} \equiv m_{A, i}$ and $b_{A} \equiv b_{A, i}$ are of order the weak scale or somewhat (an order of magnitude) larger. The normalizations for $A_{\mu}, \lambda$ and $D$ are such that the inverse squares of the 4D gauge couplings, $1 / g_{i}^{2}$, appear in front of the kinetic terms.

We assume that the parameters in Eq. (11) are real. There are two gauginos, mixtures of $\lambda$ and $\psi$, for each gauge group factor, and their masses are given by diagonalizing Eq. (11) as

$$
m_{\lambda}^{2}=\frac{1}{2}\left\{2 g^{2} m_{D}^{2}+m_{A}^{2} \pm \sqrt{4 g^{2} m_{D}^{2} m_{A}^{2}+m_{A}^{4}}\right\}
$$

where we have suppressed the index $i=1,2,3$ for $m_{\lambda}, g, m_{D}$ and $m_{A}$. The squark and slepton masses arise from finite one-loop diagrams as

$$
m_{\tilde{f}}^{2}=\sum_{i=1,2,3} \frac{g_{i}^{4} C_{i}^{\tilde{f}}}{4 \pi^{2}} \hat{M}_{i}^{2}
$$

where $\left(C_{1}^{\tilde{f}}, C_{2}^{\tilde{f}}, C_{3}^{\tilde{f}}\right)=(1 / 60,3 / 4,4 / 3),(4 / 15,0,4 / 3),(1 / 15,0,4 / 3),(3 / 20,3 / 4,0)$ and $(3 / 5,0,0)$ for $\tilde{f}=\tilde{q}, \tilde{u}, \tilde{d}, \tilde{l}$ and $\tilde{e}$, respectively, and $\hat{M}_{i}^{2}$ are given by

$$
\hat{M}_{i}^{2}=m_{D}^{2}\left\{\ln \left(\frac{4 g^{2} m_{D}^{2}+m_{A}^{2}-b_{A}+m_{a}^{2}}{g^{2} m_{D}^{2}}\right)-\frac{m_{A}}{\sqrt{4 g^{2} m_{D}^{2}+m_{A}^{2}}} \ln \left(\frac{\sqrt{4 g^{2} m_{D}^{2}+m_{A}^{2}}+m_{A}}{\sqrt{4 g^{2} m_{D}^{2}+m_{A}^{2}}-m_{A}}\right)\right\}
$$

which are positive in the entire parameter region [16]. Here, we have suppressed the index $i=1,2,3$ for $g, m_{D}, m_{A}, b_{A}$ and $m_{a}^{2}$.

As we will see later, the relevant parameter region for us is where $\tan \beta \equiv\left\langle H_{u}\right\rangle /\left\langle H_{d}\right\rangle$ is not large, e.g. $\tan \beta \lesssim 3$, so the only important Yukawa coupling is the top Yukawa coupling. The soft supersymmetry breaking masses for the Higgs doublets are then given by

$$
m_{H_{u}}^{2} \approx m_{\tilde{l}}^{2}-\frac{3 y_{t}^{2}}{8 \pi^{2}}\left(m_{\tilde{q}}^{2}+m_{\tilde{u}}^{2}\right) \ln \left(\frac{m_{D, 3}}{m_{\tilde{q}}}\right), \quad m_{H_{d}}^{2} \approx m_{\tilde{l}}^{2},
$$

where we have used the fact that the mediation scale for the squark masses is of order the Dirac gluino mass $m_{D, 3}$, and we have approximated $m_{\tilde{q}} \approx m_{\tilde{u}}$ inside the logarithm. A small soft mass squared for $S, m_{S}^{2}$, is also generated at one loop through $\lambda$, picking up $m_{H_{u}}^{2}$ and $m_{H_{d}}^{2}$. 
Equation (15) explicitly demonstrates that the effective messenger scale for this theory is very low

$$
M_{\text {mess }} \approx m_{D, 3} \approx \frac{\sqrt{3 \pi^{2}}}{g_{3}} m_{\tilde{q}}
$$

so that larger squark masses can, in principle, be obtained for a given fine-tuning and Higgs boson mass, compared with gauge-mediation-type models such as the ones considered in $[2,3]$. This is because the squark squared masses are suppressed by a one-loop factor compared with the squared messenger scale in the present theory, while they are suppressed by a two-loop factor in gauge-mediation-type models.

Weak-scale values for the couplings $\lambda$ and $\kappa$ are subject to the constraint that they do not hit the Landau pole below the unification scale. In our theory, the 321 gauge couplings are large at the ultraviolet due to the introduction of the $A$ fields and any additional fields, e.g., needed to recover coupling unification, which significantly weakens these constraints. We find that $\lambda \lesssim 0.8$ can be obtained for $\tan \beta \gtrsim 1.8$ for sufficiently large matter content, while the bound becomes somewhat stronger for smaller $\tan \beta$, e.g. $\lambda \lesssim 0.7$ for $\tan \beta \gtrsim 1.4[2,24]$. Note that, with the strong 321 gauge couplings at the ultraviolet, $\tan \beta$ as small as $\sim 1.2$ is allowed because $y_{t}$ receives a strong asymptotically non-free contribution from a large $S U(3)_{C}$ coupling at the ultraviolet. The bound on $\kappa$ is given by $\kappa \lesssim 0.2(0.3)$ for $\lambda \simeq 0.8(0.7)$.

\subsection{Suppression of the $D$-term potential and a constraint from the $\rho$ parameter}

The operators in Eq. (2) give mixings between the auxiliary $D$ fields and the scalar components of $A$ (the third and fourth terms of Eq. (11)). As a consequence, the $S U(2)_{L}$ and $U(1)_{Y} D$ term contributions to the Higgs quartic couplings are suppressed [16]. Denoting the suppression factors by $\epsilon\left(\epsilon_{2}\right.$ and $\epsilon_{1}$ for $S U(2)_{L}$ and $U(1)_{Y}$, respectively), they are given by

$$
\epsilon=\frac{m_{A}^{2}-b_{A}+m_{a}^{2}}{4 g^{2} m_{D}^{2}+m_{A}^{2}-b_{A}+m_{a}^{2}},
$$

where, again, we have suppressed the index $i=1,2$. The $D$-term contributions to the Higgs potential are given by $\epsilon$ times the standard contributions.

The suppression of the $D$-term potential can also be seen before integrating out the $A$ fields. Focusing on the $T^{3}$ direction of $S U(2)_{L}$, the corresponding $D$-term potential is given, after integrating out the $D$ field, by

$$
V=\frac{g_{2}^{2}}{2}\left(\frac{1}{2} h_{u}^{2}-\frac{1}{2} h_{d}^{2}+2 m_{D, 2} \varphi_{2}\right)^{2},
$$


where $\varphi_{2}$ is the imaginary part of the $T^{3}$ component of the $S U(2)_{L}$ adjoint field, $a_{2}=i \varphi_{2} / \sqrt{2}+$ $\cdots$, and we have retained only the components for the Higgs doublets that obtain VEVs, $H_{u}=$ $\left(0, h_{u}\right)^{T}$ and $H_{d}=\left(h_{d}, 0\right)^{T}$. The potential of Eq. (18) forces $\varphi_{2}$ to have a VEV

$$
\left\langle\varphi_{2}\right\rangle=\left(1-\epsilon_{2}\right) \frac{\cos (2 \beta)}{4 m_{D, 2}} v^{2} \approx \frac{\cos (2 \beta)}{4 m_{D, 2}} v^{2},
$$

where $v^{2} \equiv\left\langle h_{u}\right\rangle^{2}+\left\langle h_{d}\right\rangle^{2}$ and $\epsilon_{2}$ is given by Eq. (17). This mostly cancels the $D$-term potential. (We find $V=\left(\epsilon_{2} g_{2}^{2} / 2\right)\left(\left\langle h_{u}\right\rangle^{2} / 2-\left\langle h_{d}\right\rangle^{2} / 2\right)$ by substituting $\left\langle\varphi_{2}\right\rangle$ back to $V$.)

The size of any $S U(2)_{L}$ triplet $\mathrm{VEV}$ is subject to a stringent constraint from electroweak data (the $\rho$ parameter). This gives the upper bound on the value of $\left\langle\varphi_{2}\right\rangle$, and thus the lower bound on $m_{D, 2}$. Requiring $|\rho-1| \approx \cos ^{2}(2 \beta) v^{2} / 8 m_{D, 2}^{2} \lesssim 0.002$ [25], we find $m_{D, 2} \gtrsim 1 \mathrm{TeV}$ for $\tan \beta \approx 2$. This bound is easily satisfied in the parameter region considered in the next subsection.

The imaginary part of the singlet field $a_{1}$ also receives a small VEV of order $v^{2} / m_{D, 1}$, analogously to $\varphi_{2}$. This VEV, however, does not affect phenomenology except that it is responsible for the suppression of the $U(1)_{Y} D$-term potential.

\subsection{Electroweak symmetry breaking}

We are now ready to discuss electroweak symmetry breaking. Our Higgs potential is given by

$$
V=V_{F}+V_{D}+V_{\text {soft }}
$$

where $V_{F}, V_{D}$ and $V_{\text {soft }}$ are given by

$$
\begin{aligned}
V_{F} & =\left|\lambda H_{u} H_{d}+M_{S} S+\kappa S^{2}\right|^{2}+\left|\lambda S H_{u}+\mu H_{u}\right|^{2}+\left|\lambda S H_{d}+\mu H_{d}\right|^{2}, \\
V_{D} & =\epsilon_{2} \frac{g_{2}^{2}}{2} \sum_{a=1}^{3}\left(H_{u}^{\dagger} \frac{\sigma^{a}}{2} H_{u}+H_{d}^{\dagger} \frac{\sigma^{a}}{2} H_{d}\right)^{2}+\epsilon_{1} \frac{3 g_{1}^{2}}{10}\left(\frac{1}{2} H_{u}^{\dagger} H_{u}-\frac{1}{2} H_{d}^{\dagger} H_{d}\right)^{2}, \\
V_{\text {soft }} & =m_{H_{u}}^{2}\left|H_{u}\right|^{2}+m_{H_{d}}^{2}\left|H_{d}\right|^{2}+\left(b_{H} H_{u} H_{d}+\frac{b_{S}}{2} S^{2}+\text { h.c. }\right) .
\end{aligned}
$$

Here, $\epsilon_{2}$ and $\epsilon_{1}$ are given by Eq. (17), and $m_{H_{u}}^{2}$ and $m_{H_{d}}^{2}$ by Eq. (15). The holomorphic supersymmetry breaking masses $b_{H}$ and $b_{S}$, which we write as

$$
\begin{aligned}
b_{H} & =\mu m_{3 / 2}-b_{H, 0}, \\
b_{S} & =M_{S} m_{3 / 2}-b_{S, 0},
\end{aligned}
$$

are given by Eq. (10). Other supersymmetry breaking parameters are also generated at higher loop orders. 


\begin{tabular}{|c|c|c|c|c|}
\hline & $\mathrm{A}$ & $\mathrm{B}$ & $\mathrm{C}$ & $\mathrm{D}$ \\
\hline$\lambda$ & 0.8 & 0.8 & 0.7 & 0.7 \\
$\kappa$ & 0.2 & 0.2 & 0.2 & 0.2 \\
$\mu$ & 177 & 226 & 186 & 236 \\
$M_{S}$ & 355 & 452 & 373 & 473 \\
$m_{3 / 2}$ & 183 & 234 & 225 & 285 \\
{$\left[b_{H, 0}\right]^{1 / 2}$} & -100 & -93 & 41 & -111 \\
{$\left[b_{S, 0}\right]^{1 / 2}$} & -133 & -177 & -86 & -110 \\
\hline$m_{D, 1}$ & 5076 & 6935 & 5331 & 8112 \\
$m_{D, 2}$ & 2201 & 2688 & 2312 & 3424 \\
$m_{D, 3}$ & 2018 & 2571 & 2120 & 2690 \\
$m_{A, 1}$ & 385 & 491 & 2242 & 2853 \\
$m_{A, 2}$ & 269 & 343 & 2415 & 2527 \\
$m_{A, 3}$ & 459 & 584 & 2312 & 2935 \\
{$\left[b_{A, 1}\right]^{1 / 2}$} & 92 & 117 & 225 & 285 \\
{$\left[b_{A, 2}\right]^{1 / 2}$} & 110 & 140 & 308 & 391 \\
{$\left[b_{A, 3}\right]^{1 / 2}$} & 318 & 405 & 334 & 424 \\
\hline tan $\beta$ & 1.8 & 1.8 & 1.7 & 1.6 \\
$\mu_{\text {eff }}$ & 175 & 224 & 185 & 236 \\
{$\left[(\mu)_{\text {eff }}\right]^{1 / 2}$} & 204 & 246 & 199 & 282 \\
{$\left[m_{H_{u}}^{2}\right]^{1 / 2}$} & -123 & -159 & -137 & -117 \\
{$\left[m_{H_{d}}^{2}\right]^{1 / 2}$} & 170 & 213 & 153 & 244 \\
$M_{\text {Higgs }}$ & 134 & 138 & 128 & 135 \\
\hline$\langle S\rangle$ & -2.8 & -2.4 & -2.1 & -1.3 \\
$\epsilon_{1}$ & 0.0063 & 0.0054 & 0.17 & 0.12 \\
$\epsilon_{2}$ & 0.0073 & 0.0080 & 0.39 & 0.24 \\
\hline$\left(m_{\tilde{q}}^{2}\right)^{1 / 2}$ & 538 & 683 & 519 & 672 \\
$\left(m_{\tilde{u}}^{2}\right)^{1 / 2}$ & 527 & 673 & 512 & 657 \\
$\left(m_{\tilde{d}}^{2}\right)^{1 / 2}$ & 519 & 661 & 504 & 642 \\
$\left(m_{\tilde{l}}^{2}\right)^{1 / 2}$ & 170 & 213 & 153 & 244 \\
$\left(m_{\tilde{e}}^{2}\right)^{1 / 2}$ & 159 & 217 & 156 & 242 \\
\hline$\tilde{\Delta}^{-1}$ & $24 \%$ & $16 \%$ & $20 \%$ & $13 \%$ \\
\hline & & & & \\
\hline
\end{tabular}

Table 2: Values for the parameters of the model for four sample points, A, B, C and D. The resulting soft supersymmetry breaking masses for squarks and sleptons as well as the quantities in the Higgs sector are also listed. Here, $[X]^{n} \equiv \operatorname{sgn}(X) \cdot|X|^{n}$, and all masses are given in units of $\mathrm{GeV}$. The fine-tuning parameter $\tilde{\Delta}^{-1}$ is defined in Ref. [2]. 
The results of the potential minimization are given in Table 2 for four sample points A, $\mathrm{B}, \mathrm{C}$ and $\mathrm{D}$, which lead to realistic phenomenology. The parameters $m_{D, i}, m_{A, i}$, and $b_{A, i}$ $(i=1,2,3)$ are Dirac gaugino masses, supersymmetric masses for $A_{i}$, and holomorphic supersymmetry breaking masses for $A_{i}$, respectively, and defined below Eq. (7), below Eq. (3), and in Eq. (6). The square bracket in the table is defined as $[X]^{n} \equiv \operatorname{sgn}(X) \cdot|X|^{n}$, and all masses are given in units of $\mathrm{GeV}$. The effective $\mu$ and $\mu B$ parameters are defined by $\mu_{\mathrm{eff}} \equiv \mu+\lambda\langle S\rangle$ and $(\mu B)_{\text {eff }} \equiv b_{H}+\lambda\left(M_{S}\langle S\rangle+\kappa\langle S\rangle^{2}\right)$, and $M_{\text {Higgs }}$ is the lightest Higgs boson mass. We also list the parameter $\tilde{\Delta}^{-1}$ defined in Ref. [2], following [26], as a measure of fine-tuning in our theory. All the parameters in the Higgs potential are taken to be real.

Our procedure to obtain these numbers is as follows. The input parameters of the analysis are $\lambda, \kappa, \mu, M_{S}, m_{3 / 2}, b_{H, 0}, b_{S, 0}, m_{D, i}, m_{A, i}$, and $b_{A, i}$. Using these, we can derive $m_{H_{u}}^{2}$ and $m_{H_{d}}^{2}$, assuming some initial value for $\tan \beta$ (which will be determined in the end by iteration). This determines the Higgs potential of Eq. (20). We also add the one-loop contribution from top-stop loops to the Higgs quartic coupling in our analysis. (A precise calculation of this contribution requires a knowledge of $\langle S\rangle$, determined by iteration, but the effect of $\langle S\rangle \neq 0$ is negligible.) Corrections from higher loops are not so large for the values of top squark masses considered here, only giving an additional negative contribution to the lightest Higgs boson mass of order a few GeV. By minimizing the potential, we obtain $\left\langle H_{u}\right\rangle,\left\langle H_{d}\right\rangle$ and $\langle S\rangle$. These VEVs do not in general satisfy $v_{H}^{2} \equiv\left\langle H_{u}\right\rangle^{2}+\left\langle H_{d}\right\rangle^{2}=(174 \mathrm{GeV})^{2}$, so we iterate the entire process again using the input parameters appropriately rescaled by powers of $\left(174 \mathrm{GeV} / v_{H}\right)$ according to their dimensions. In this process we use the derived value of $\tan \beta, \tan \beta=\left\langle H_{u}\right\rangle /\left\langle H_{d}\right\rangle$ to determine $m_{H_{u}}^{2}$. By iterating this several times, we obtain the final values for the parameters, which gives $\left\langle H_{u}\right\rangle^{2}+\left\langle H_{d}\right\rangle^{2}=(174 \mathrm{GeV})^{2}$. The convergence of the whole procedure is fairly quick.

As is seen in the table, the fine-tuning required in our theory is very mild and only of order $20 \%$. We find that the electroweak scale $v$ is mainly sensitive to the values of $\mu, m_{3 / 2}, m_{D, 2}$, $m_{D, 3}, g_{2}, g_{3}$ and $y_{t}$, and the fine-tuning parameter is determined by the sensitivity to $\mu, m_{3 / 2}$, $m_{D, 3}, g_{3}$ and $y_{t}$ in most of the parameter region. The reduction of the fine-tuning occurs mainly because the restoring force of the Higgs potential arises from the F-term potential, which is stronger than the one from the $S U(2) \times U(1)_{Y} D$-term potential [18]. A very small effective messenger scale of Eq. (16) then allows squark masses as large as $(500 \sim 700) \mathrm{GeV}$. As can be seen from the table, electroweak symmetry breaking in our theory is caused by an interplay between the $\mu B$ term and the top-stop radiative correction to $m_{H_{u}}^{2}$ - the diagonal entries in the Higgs boson mass-squared matrix, $\approx\left\{\left|\mu_{\mathrm{eff}}\right|^{2}+m_{H_{u}}^{2},\left|\mu_{\mathrm{eff}}\right|^{2}+m_{H_{d}}^{2}\right\}$, are both positive, and one of the eigenvalues becomes negative because of a non-zero value of $(\mu B)_{\mathrm{eff}}$. 


\subsection{Superparticle, Higgs boson and adjoint scalar spectrum}

The masses for the superparticles, the Higgs bosons, and the adjoint scalars are calculated at tree level for the four sample points in Table 2, which are listed in Table 3. The $\tilde{g}_{1,2}, \chi_{1,2,3}^{ \pm}$, and $\chi_{1-7}^{0}$ represent the two gluinos, three charginos, and seven neutralinos, respectively, which come from the linear combinations of the original gauginos, $\lambda_{i}^{\alpha}$, and the fermionic components of $A_{i}$, $H_{u}$ and $H_{d}$. The $\tilde{u}_{L, R}, \tilde{d}_{L, R}, \tilde{e}_{L, R}$, and $\tilde{\nu}_{L}$ represent the left- and right-handed up-type squarks, down-type squarks, charged sleptons, and the (left-handed) sneutrinos, respectively. The masses for the top squarks, $\tilde{t}_{1,2}$, are listed separately because they split from the other squark masses appreciably. The neutral scalar, pseudo-scalar, and charged Higgs bosons are labeled as $H_{1,2,3}^{0}$, $P_{1,2}^{0}$, and $H^{ \pm}$, respectively, which arise from the scalar components of $S, H_{u}$ and $H_{d}$. There are two adjoint scalar fields for each gauge group factor, which are denoted by $a_{Y}, a_{L}$, and $a_{C}$ for $U(1)_{Y}, S U(2)_{L}$, and $S U(3)_{C}$, respectively. The gravitino is denoted by $\tilde{G}$.

In the first two points, $\mathrm{A}$ and $\mathrm{B}$, the parameters are chosen such that $m_{A, i} \ll m_{D, i}$, so that the two gauginos for each gauge group factor are relatively close in masses: $\left\{\tilde{g}_{1}, \tilde{g}_{2}\right\}$ for $S U(3)_{C}$, $\left\{\chi_{2}^{ \pm}, \chi_{3}^{ \pm}\right\}$and $\left\{\chi_{4}^{0}, \chi_{5}^{0}\right\}$ for $S U(2)_{L}$, and $\left\{\chi_{6}^{0}, \chi_{7}^{0}\right\}$ for $U(1)_{Y}$. Because of small values for $m_{A, i}$, one of the two adjoint scalars for each gauge group factor, $a_{Y, 1}, a_{L, 1}$ and $a_{C, 1}$, are relatively light (below a TeV). For the other two points, $\mathrm{C}$ and $\mathrm{D}$, the parameters are chosen such that $m_{A, i} \sim m_{D, i}$. Thus the two gaugino masses are not necessarily close, and the adjoint scalars are all heavy, with masses above $2 \mathrm{TeV}$.

An interesting feature of the present model is that the effects of the gauge $D$-terms are suppressed because of mixings between the auxiliary $D$ fields and the $a$ fields (see Eq. (11)). This also affects the spectrum of superparticles. For points A and B, the $S U(2)_{L}$ and $U(1)_{Y}$ $D$-terms receive large suppressions, $\epsilon_{1}, \epsilon_{2} \ll 1$ (see Table 2). As a consequence, the squarks and sleptons that are in the same $S U(2)_{L}$ multiplet are almost completely degenerate in mass. (Mass splittings of order a few hundreds of $\mathrm{MeV}$ are generated from radiative corrections.) For points $\mathrm{C}$ and $\mathrm{D}$, the suppressions are not as strong as the case of points $\mathrm{A}$ and $\mathrm{B}$, because of relatively large values of $m_{A, i}$, but the squarks and sleptons in the same $S U(2)_{L}$ multiplet are still quite degenerate.

We find that the lightest supersymmetric particle (LSP) in our theory can either be the third generation right-handed slepton $\tilde{e}_{R}$, the third generation sneutrino $\tilde{\nu}_{L}$, the lightest neutralino

$\chi_{1}^{0}$, or the gravitino $\tilde{G}$. In either case, the mass of the LSP is naturally in the range $\approx(100 \sim$ $300) \mathrm{GeV}$. Because of rather small values for $\tan \beta$ and $M_{\text {mess }}$, the masses of the third generation $\tilde{e}_{R}$ and $\tilde{\nu}_{L}$ are almost degenerate with those of the corresponding first-two generation particles. For the case of the $\tilde{\nu}_{L}$ LSP, the left-handed selectrons $\tilde{e}_{L}$ will also be very close in mass, with the mass difference to $\tilde{\nu}_{L}$ only of order a few hundreds of $\mathrm{MeV}$ to a few $\mathrm{GeV}$. For the $\chi_{1}^{0} \mathrm{LSP}$, it is almost purely the Higgsino, so that the lightest chargino $\chi_{1}^{ \pm}$will be close in mass to $\chi_{1}^{0}$ with 


\begin{tabular}{|c|c|c|c|c|}
\hline & $\mathrm{A}$ & $\mathrm{B}$ & $\mathrm{C}$ & $\mathrm{D}$ \\
\hline$\tilde{g}_{1}$ & 1982 & 2526 & 1427 & 1812 \\
$\tilde{g}_{2}$ & 2441 & 3110 & 3740 & 4747 \\
\hline$\chi_{1}^{ \pm}$ & 175 & 224 & 189 & 238 \\
$\chi_{2}^{ \pm}$ & 1305 & 1586 & 726 & 1299 \\
$\chi_{3}^{ \pm}$ & 1574 & 1929 & 3136 & 3823 \\
\hline$\chi_{1}^{0}$ & 169 & 219 & 186 & 236 \\
$\chi_{2}^{0}$ & 207 & 250 & 208 & 255 \\
$\chi_{3}^{0}$ & 392 & 482 & 400 & 494 \\
$\chi_{4}^{0}$ & 1305 & 1586 & 726 & 1299 \\
$\chi_{5}^{0}$ & 1574 & 1929 & 1599 & 2605 \\
$\chi_{6}^{0}$ & 2175 & 2987 & 3136 & 3823 \\
$\chi_{7}^{0}$ & 2560 & 3478 & 3840 & 5458 \\
\hline$\tilde{u}_{L}$ & 538 & 683 & 519 & 672 \\
$\tilde{u}_{R}$ & 527 & 673 & 512 & 657 \\
$\tilde{d}_{L}$ & 538 & 683 & 520 & 672 \\
$\tilde{d}_{R}$ & 519 & 661 & 504 & 642 \\
$\tilde{e}_{L}$ & 170 & 213 & 155 & 244 \\
$\tilde{e}_{R}$ & 159 & 217 & 157 & 242 \\
$\tilde{\nu}_{L}$ & 170 & 213 & 151 & 243 \\
\hline$\tilde{t}_{1}$ & 521 & 654 & 505 & 634 \\
$\tilde{t}_{2}$ & 561 & 696 & 545 & 686 \\
\hline$H_{1}^{0}$ & 134 & 138 & 128 & 135 \\
$H_{2}^{0}$ & 285 & 357 & 284 & 405 \\
$H_{3}^{0}$ & 476 & 600 & 493 & 618 \\
$P_{1}^{0}$ & 173 & 220 & 177 & 253 \\
$P_{2}^{0}$ & 365 & 430 & 354 & 453 \\
$H^{ \pm}$ & 280 & 353 & 281 & 403 \\
\hline$a_{Y, 1}$ & 396 & 505 & 2253 & 2868 \\
$a_{Y, 2}$ & 4733 & 6464 & 5434 & 8057 \\
$a_{L, 1}$ & 291 & 370 & 2435 & 2557 \\
$a_{L, 2}$ & 2872 & 3509 & 3844 & 5104 \\
$a_{C, 1}$ & 558 & 711 & 2336 & 2965 \\
$a_{C, 2}$ & 4412 & 5622 & 5156 & 6544 \\
\hline$\tilde{G}^{0}$ & 183 & 234 & 225 & 285 \\
\hline
\end{tabular}

Table 3: The masses for the superparticles, Higgs bosons and adjoint scalars for the four sample points A, B, C and D given in Table 2. All masses are given in units of GeV. 
the mass difference of order a few $\mathrm{GeV}$.

The lightest Higgs boson in our theory cannot be heavier than about $140 \mathrm{GeV}$. The mass of the charged Higgs boson is also bounded by $m_{H^{ \pm}} \lesssim 450 \mathrm{GeV}$, as the amount of fine-tuning is correlated with the charged Higgs boson mass [18]. This may have some implications on the rate of the $b \rightarrow s \gamma$ process. While the current theoretical estimates for this process still have some uncertainties [27], the positive sign for the effective $\mu$ parameter seems to be preferred over the other one, with which a partial cancellation between the charged Higgs boson and chargino contributions is possible.

\section{Models with Sequestered Gauge Mediation}

In this section we present the second class of models. We find that the fine-tuning is reduced to the level of $(10 \sim 20) \%$ in these models.

\subsection{Models}

Our basic idea here is the following. We consider gauge mediation models, in which superparticle masses are generated by loops of messenger fields [28, 29]. In particular, we consider a model in which supersymmetric and supersymmetry breaking masses for the messenger fields do not possess any particular "unified" relations (this requires multiple singlets in the messenger sector) $[2,30]$. Now, suppose that all the MSSM fields together with the messenger fields are localized on a $(3+1)$-dimensional subspace in some higher dimensional spacetime, and that supersymmetry breaking occurs at some other subspace, which is transmitted to the messenger sector through some bulk interactions. In this case, we can push up the fundamental scale of supersymmetry breaking to an intermediate scale without affecting the gauge mediated spectrum for the MSSM superparticles. On the other hand, supersymmetric masses of order the weak scale can be generated in the Higgs sector from the Kähler potential terms, as was the case in section 2.3. In fact, this structure was used in Ref. [17] to generate the $\mu$ term in gauge mediation models, where the coincidence of the scales for the $\mu$ term and for the superparticle masses was also naturally obtained. Here we adopt the basic construction of this model to demonstrate our point.

Let us consider $(4+1)$-dimensional spacetime with the extra dimension compactified on an

$S^{1} / Z_{2}$ orbifold, $y:[0,2 \pi]$, where $y$ is the coordinate for the fifth dimension. The size of the extra dimension we consider is small, only one or two orders of magnitude larger than the inverse of the fundamental scale, which is of order the Planck scale. We consider that supersymmetry is dynamically broken on the $y=\pi R$ brane at the scale $\Lambda$, and (some of) the fields participating in this dynamics are charged under a $U(1)_{m}$ gauge multiplet located in the bulk [17]. Our messenger 
sector is localized on the $y=0$ brane. Let us first consider only a single vector-like messenger $\mathcal{D}\left(\mathbf{3}^{*}, \mathbf{1}\right)_{1 / 3}+\overline{\mathcal{D}}(\mathbf{3}, \mathbf{1})_{-1 / 3}$, where the numbers represent the 321 gauge quantum numbers. The superpotential interactions in the messenger sector are then given by

$$
\mathcal{L}=\delta(y) \int d^{2} \theta\left(k_{E} X E \bar{E}+\frac{f}{3} X^{3}+k_{\mathcal{D}} X \mathcal{D} \overline{\mathcal{D}}\right)+\text { h.c. },
$$

where $X, E$ and $\bar{E}$ are singlets under 321 , and the $U(1)_{m}$ charges for these fields are chosen as $E(+1), \bar{E}(-1), X(0), \mathcal{D}(0)$ and $\overline{\mathcal{D}}(0)$. Supersymmetry breaking is mediated from the $y=\pi R$ brane to the $y=0$ brane through $U(1)_{m}$ gauge interactions, generating positive supersymmetry breaking squared masses of order $\approx\left(g_{m}^{2} / 16 \pi^{2}\right)^{2} \Lambda^{2}$ for $E$ and $\bar{E}$. Here, $g_{m}$ is the $4 \mathrm{D} U(1)_{m}$ gauge coupling, which is naturally suppressed by the volume of the extra dimension. These positive squared masses in turn generate a negative mass squared for $X$ through the coupling $k_{E}$, triggering the VEVs for the lowest and highest components of the $X$ chiral superfield: $\langle X\rangle \neq 0$ and $\left\langle F_{X}\right\rangle \neq 0$. Note that, while the superpotential interactions of Eq. (26) possess a $U(1)_{R}$ symmetry, it is explicitly broken by the trilinear scalar interactions arising from anomaly mediation [23, 31], so that the dangerous Goldstone boson does not arise. These VEVs then provide the supersymmetric and supersymmetry breaking masses for the messenger fields $\mathcal{D}$ and $\overline{\mathcal{D}}: M_{\mathcal{D}}=k_{\mathcal{D}}\langle X\rangle$ and $F_{\mathcal{D}}=k_{\mathcal{D}}\left\langle F_{X}\right\rangle$. For $k_{E} \sim f \sim k_{\mathcal{D}} \sim O(1)$, the sizes of these masses are $M_{\mathcal{D}}^{2} \sim F_{\mathcal{D}} \sim O\left(g_{m}^{4} \Lambda^{2} /\left(16 \pi^{2}\right)^{3}\right)$, so that we can naturally obtain $M_{\mathcal{D}} / \Lambda \sim \sqrt{F_{\mathcal{D}}} / \Lambda \sim\left(10^{-6} \sim 10^{-5}\right)$ for $g_{m}^{2} \approx\left(10^{-3} \sim 10^{-2}\right)$, which is consistent with the volume suppression of $g_{m}$. We thus take $\Lambda \approx 10^{10} \mathrm{GeV}$ and $M_{\mathcal{D}} \approx \sqrt{F_{\mathcal{D}}} \approx(10 \sim 100) \mathrm{TeV}$ in our analysis. As we have seen, this requires some coincidence of the scales but does not require fine-tuning.

Our messenger sector also contains vector-like messenger fields other than $\mathcal{D}$ and $\overline{\mathcal{D}}$. In particular, to preserve the successful prediction for gauge coupling unification at the leading-log level, we introduce messenger fields in complete $S U(5)$ multiplets. Specifically, we introduce $n_{\mathbf{5}}$ pairs of $(\mathcal{D}, \mathcal{L})+(\overline{\mathcal{D}}, \overline{\mathcal{L}})$ and $n_{\mathbf{1 0}}$ pairs of $(\mathcal{Q}, \mathcal{U}, \mathcal{E})+(\overline{\mathcal{Q}}, \overline{\mathcal{U}}, \overline{\mathcal{E}})$, where the 321 gauge quantum numbers of $\mathcal{Q}, \mathcal{U}, \mathcal{D}, \mathcal{L}$ and $\mathcal{E}$ are the same as the corresponding MSSM fields. The numbers $n_{5}$ and $n_{10}$ are bounded by $n_{5}+3 n_{10} \lesssim 5$ due to the Landau pole consideration for the 321 gauge couplings. We consider that each component of the messenger fields has independent supersymmetric and supersymmetry breaking masses $M$ and $F$ : for example, we treat $M_{\mathcal{D}}, F_{\mathcal{D}}$, $M_{\mathcal{L}}$ and $F_{\mathcal{L}}$ to be all independent for $\left(n_{\mathbf{5}}, n_{\mathbf{1 0}}\right)=(1,0)$. There are a number of ways to achieve this. The easiest way is to introduce $E, \bar{E}$ and $X$ fields as well as the interactions of Eq. (26) for each messenger field. Such a structure can naturally arise if we introduce a discrete $Z_{3}$ symmetry for each component of the messenger fields. ${ }^{7}$ In any event, with these most general $M$ 's and $F$ 's,

\footnotetext{
${ }^{7}$ These structures are consistent with gauge unification if the unified symmetry is realized in higher dimensions [32].
} 
the gaugino masses, $M_{a}$, and the sfermion masses, $m_{\tilde{f}}$, at the messenger scale are written as

$$
M_{i}=\frac{g_{i}^{2}}{16 \pi^{2}} \Lambda_{G, i}
$$

and

$$
m_{\tilde{f}}^{2}=2 \sum_{i=1,2,3}\left(\frac{g_{i}^{2}}{16 \pi^{2}}\right)^{2} C_{i}^{\tilde{f}} \Lambda_{S, i}^{2},
$$

where $i=1,2,3$ represents $U(1)_{Y}, S U(2)_{L}$ and $S U(3)_{C}$, and $C_{i}^{\tilde{f}}$ are the group theoretical factors. The parameters $\Lambda_{G, i}$ and $\Lambda_{S, i}$ are of order $(10 \sim 100) \mathrm{TeV}$, which can be explicitly calculated in terms of the $M$ 's and $F$ 's once the field content for the messengers is specified.

The Higgs sector of the present model is essentially the same as the one in the previous model (see section 2.3). The field content is given by $S(\mathbf{1}, \mathbf{1})_{0}, H_{u}(\mathbf{1}, \mathbf{2})_{1 / 2}$ and $H_{d}(\mathbf{1}, \mathbf{2})_{-1 / 2}$. Imposing the discrete $Z_{4, R}$ symmetry of Table 1 , the effective superpotential arises both from $W_{0}=\lambda S H_{u} H_{d}+(\kappa / 3) S^{3}$ and $K=\lambda_{H} H_{u} H_{d}+\left(\lambda_{S} / 2\right) S^{2}+$ h.c. as

$$
W_{H}=\lambda S H_{u} H_{d}+\mu H_{u} H_{d}+\frac{M_{S}}{2} S^{2}+\frac{\kappa}{3} S^{3},
$$

where $\mu$ and $M_{S}$ are parameters of order the weak scale generated via the mechanism of [15]. Note that this mechanism works even if the supersymmetry breaking sector (at the $y=\pi R$ brane) and the Higgs sector (at the $y=0$ brane) are geometrically separated [17]. The holomorphic supersymmetry breaking terms

$$
\mathcal{L}_{H, \text { soft }} \equiv-b_{H} H_{u} H_{d}-\frac{b_{S}}{2} S^{2}+\text { h.c. }
$$

are also generated from the Kähler potential terms as

$$
\begin{aligned}
b_{H} & =\mu m_{3 / 2}, \\
b_{S} & =M_{S} m_{3 / 2},
\end{aligned}
$$

where $m_{3 / 2} \approx \Lambda^{2} / M_{\mathrm{Pl}}$ is the gravitino mass of order the weak scale. The Yukawa couplings for the quark and lepton superfields are given by $W=y_{u} Q U H_{u}+y_{d} Q D H_{d}+y_{e} L E H_{d}$.

We here note that a theory having essentially the same properties can also be formulated in warped spacetime of [33]. We can simply make our $S^{1} / Z_{2}$ extra dimension warped, with the scales on the ultraviolet and infrared branes set to be around the 4D Planck scale and the intermediate scale, respectively. The MSSM fields, the singlet field $S$, and fields in the messenger sector are all localized on the ultraviolet brane, while the $U(1)_{m}$ gauge multiplet propagates in the bulk. Supersymmetry breaking occurs on the infrared brane, which is transmitted to the $E$ and $\bar{E}$ fields on the ultraviolet brane through bulk $U(1)_{m}$ gauge interactions, as in the models of $[34,35]$. This theory allows a purely 4D interpretation through the AdS/CFT correspondence [36, 37], in which the separation of supersymmetry breaking and the other fields occurs through conformal sequestering effects [38]. 


\subsection{Electroweak symmetry breaking and particle spectrum}

Now we study electroweak symmetry breaking in our model. The Higgs potential is given by Eq. (20) with Eqs. $(21-23)$, but with both $\epsilon_{1}$ and $\epsilon_{2}$ set to 1 in Eq. (22). The holomorphic supersymmetry breaking masses, $b_{H}$ and $b_{S}$, are given by Eqs. $(31,32)$ rather than Eqs. $(24,25)$. For smaller number of messenger fields, the 321 gauge couplings are not very strong at the unification scale, so that the value of $\lambda$ should be somewhat smaller than 0.8 to avoid the Landau pole.

The results of the potential minimization are given in Table 4 for three sample points A, $\mathrm{B}$ and $\mathrm{C}$, which lead to realistic phenomenology. The square bracket in the table is defined as $[X]^{n} \equiv \operatorname{sgn}(X) \cdot|X|^{n}$, and all masses are given in units of $\mathrm{GeV}$ except for $M_{\text {mess }}, \Lambda_{G, i}$ and $\Lambda_{S, i}$, which are given in units of $\mathrm{TeV}$. The parameters $\Lambda_{G, i}$ and $\Lambda_{S, i}$ are defined in Eqs. $(27,28)$. The quantity $M_{\text {mess }}$ represents the scale at which the gaugino and sfermion masses of Eqs. $(27,28)$ are given, which we take as a single scale of order $\Lambda$ 's for simplicity. The sensitivity of physical quantities to this parameter is rather weak. The effective $\mu$ and $\mu B$ parameters are defined by $\mu_{\text {eff }} \equiv \mu+\lambda\langle S\rangle$ and $(\mu B)_{\text {eff }} \equiv b_{H}+\lambda\left(M_{S}\langle S\rangle+\kappa\langle S\rangle^{2}\right)$, and $M_{\text {Higgs }}$ is the lightest Higgs boson mass. We also list the fine-tuning parameter $\tilde{\Delta}^{-1}$ defined in [2]. All the parameters in the Higgs potential are taken to be real. The procedure to obtain these numbers is analogous to that in section 2.6.

As is seen in the table, we find that the fine-tuning in this theory is at the level of $(10 \sim 20) \%$. A difference from the previous model is that the logarithm $\ln \left(M_{\text {mess }} / m_{\tilde{t}}\right)$ appearing in the topstop correction to the Higgs mass-squared parameter is now not as small as the previous one. ( $M_{\text {mess }}$ is several tens of $\mathrm{TeV}$ in the present model while it is a few $\mathrm{TeV}$ in the previous model.) We find that for most of the parameter region the total mass-squared parameters for the up-type and down-type Higgs doublets are both positive, and electroweak symmetry breaking is triggered by a nonzero value of the effective $\mu B$ term, although the point $\mathrm{C}$ has a negative squared mass for the up-type Higgs field, $\mu_{\text {eff }}^{2}+m_{H_{u}}^{2}<0$. While the reduction of fine-tuning in the present model is not as large as the previous one, the situation is still much better than in conventional models of supersymmetry breaking, which typically require fine-tuning of order a few percent or even worse.

We have listed the masses for the superparticles and the Higgs bosons in Table 5 for the three sample points of Table 4. Here, we have included one-loop threshold corrections to obtain these masses, as they are relevant for colored particles especially if the masses are close to the experimental bounds. The meaning of the symbols is the same as that in Table 5: $\tilde{g}, \chi_{1,2}^{ \pm}$ and $\chi_{1-5}^{0}$ denote the gluino, charginos and neutralinos, respectively, $\tilde{u}_{L, R}, \tilde{d}_{L, R}, \tilde{e}_{L, R}$ and $\tilde{\nu}_{L}$ the squarks and sleptons, and $H_{1,2,3}^{0}, P_{1,2}^{0}$ and $H^{ \pm}$the neutral-scalar, pseudo-scalar, and charged Higgs bosons, respectively. The top squarks, $\tilde{t}_{1,2}$, are listed separately, and $\tilde{G}$ is the gravitino. 


\begin{tabular}{|c|c|c|c|}
\hline & $\mathrm{A}$ & $\mathrm{B}$ & $\mathrm{C}$ \\
\hline$\lambda$ & 0.65 & 0.75 & 0.75 \\
$\kappa$ & 0.2 & 0.2 & 0.2 \\
$\mu$ & 202 & 135 & 175 \\
$M_{S}$ & 598 & 580 & 616 \\
$m_{3 / 2}$ & 268 & 362 & 127 \\
\hline$n_{\mathbf{5}}$ & 1 & 4 & 1 \\
$n_{\mathbf{1 0}}$ & 0 & 0 & 1 \\
$M_{\text {mess }}$ & 50 & 50 & 50 \\
$\Lambda_{G, 1}$ & 53 & 100 & 150 \\
$\Lambda_{G, 2}$ & 68 & 140 & 82 \\
$\Lambda_{G, 3}$ & 30 & 39 & 48 \\
$\Lambda_{S, 1}$ & 56 & 57 & 98 \\
$\Lambda_{S, 2}$ & 68 & 72 & 49 \\
$\Lambda_{S, 3}$ & 30 & 19 & 24 \\
\hline $\tan \beta$ & 1.9 & 1.8 & 3.6 \\
$\mu_{\text {eff }}$ & 204 & 139 & 175 \\
{$\left[(\mu B)_{\text {eff }}\right]^{1 / 2}$} & 234 & 224 & 149 \\
{$\left[m_{H_{u}}^{2}\right]^{1 / 2}$} & -143 & 17 & -177 \\
{$\left[m_{H_{d}}^{2}\right]^{1 / 2}$} & 238 & 250 & 187 \\
$\langle S\rangle$ & 1.6 & 4.4 & -0.7 \\
$M_{\text {Higgs }}$ & 123 & 129 & 120 \\
\hline$M_{1}$ & 74 & 142 & 213 \\
$M_{2}$ & 185 & 391 & 224 \\
$M_{3}$ & 258 & 333 & 411 \\
$\left(m_{\tilde{\tilde{q}}}^{2}\right)^{1 / 2}$ & 450 & 406 & 434 \\
$\left(m_{\tilde{\tilde{c}}}^{2}\right)^{1 / 2}$ & 391 & 335 & 416 \\
$\left(m_{\tilde{d}}^{2}\right)^{1 / 2}$ & 387 & 331 & 406 \\
$\left(m_{\tilde{I}}^{2}\right)^{1 / 2}$ & 238 & 250 & 187 \\
$\left(m_{\tilde{\tilde{e}}}^{2}\right)^{1 / 2}$ & 94 & 95 & 165 \\
\hline$\Delta^{-1}$ & $13 \%$ & $19 \%$ & $12 \%$ \\
\hline & & &
\end{tabular}

Table 4: Values for the parameters of the model for three sample points, A, B and C. The resulting soft supersymmetry breaking masses for the gauginos, squarks and sleptons, as well as the quantities in the Higgs sector, are also listed. Here, $[X]^{n} \equiv \operatorname{sgn}(X) \cdot|X|^{n}$. All masses are given in units of $\mathrm{GeV}$ except for $M_{\text {mess }}, \Lambda_{G, i}$ and $\Lambda_{S, i}(i=1,2,3)$, which are given in units of $\mathrm{TeV}$. The fine-tuning parameter $\tilde{\Delta}^{-1}$ is defined in Ref. [2]. 


\begin{tabular}{|c|c|c|c|}
\hline & $\mathrm{A}$ & $\mathrm{B}$ & $\mathrm{C}$ \\
\hline$\tilde{g}$ & 306 & 376 & 450 \\
\hline$\chi_{1}^{ \pm}$ & 183 & 148 & 165 \\
$\chi_{2}^{ \pm}$ & 235 & 404 & 257 \\
\hline$\chi_{1}^{0}$ & 84 & 158 & 187 \\
$\chi_{2}^{0}$ & 204 & 158 & 207 \\
$\chi_{3}^{0}$ & 204 & 159 & 207 \\
$\chi_{4}^{0}$ & 216 & 365 & 216 \\
$\chi_{5}^{0}$ & 616 & 606 & 641 \\
\hline$\tilde{u}_{L}$ & 448 & 404 & 431 \\
$\tilde{u}_{R}$ & 390 & 334 & 415 \\
$\tilde{d}_{L}$ & 452 & 408 & 438 \\
$\tilde{d}_{R}$ & 388 & 331 & 407 \\
$\tilde{e}_{L}$ & 240 & 253 & 192 \\
$\tilde{e}_{R}$ & 100 & 100 & 170 \\
$\tilde{\nu}_{L}$ & 232 & 246 & 178 \\
\hline$\tilde{t}_{1}$ & 358 & 310 & 384 \\
$\tilde{t}_{2}$ & 451 & 415 & 448 \\
\hline$H_{1}^{0}$ & 123 & 129 & 120 \\
$H_{2}^{0}$ & 358 & 335 & 265 \\
$H_{3}^{0}$ & 730 & 754 & 695 \\
$P_{1}^{0}$ & 309 & 239 & 243 \\
$P_{2}^{0}$ & 503 & 460 & 588 \\
$H^{ \pm}$ & 358 & 332 & 271 \\
\hline$\tilde{G}^{0}$ & 268 & 362 & 127 \\
\hline
\end{tabular}

Table 5: The masses for the superparticles and the Higgs bosons for the three sample points A, $\mathrm{B}$ and $\mathrm{C}$ given in Table 4. All masses are given in units of $\mathrm{GeV}$. 
As is seen in the table, the theory accommodates various possibilities for the LSP. We find that the LSP in this theory can be either the lightest neutralino $\chi_{1}^{0}$, the lightest chargino $\chi_{1}^{ \pm}$, the third generation right-handed slepton $\tilde{e}_{R}$, the third generation sneutrino $\tilde{\nu}_{L}$, or the gravitino $\tilde{G}$. We note that the theory allows the relative signs between $\Lambda_{G, i}$ 's to be negative, although we did not adopt such a case in our sample points A, B and C. In the case that $\chi_{1}^{0}$ is the LSP, its thermal relics may provide the dark matter of the universe [39]. In general, the neutral LSP's, $\chi_{1}^{0}$ and $\tilde{G}$, can be the dark matter if they are nonthermally produced. The cases with charged LSP's require "non-standard" cosmology or small $R$-parity violation.

\section{Conclusions}

We have constructed two classes of realistic supersymmetric models in which no significant finetuning is required to reproduce the correct scale for electroweak symmetry breaking. Both classes of models accomplish this by incorporating (i) a means to increase the effective Higgs quartic coupling beyond what is available in the MSSM, (ii) a low scale for the generation of squark and slepton masses, and (iii) a degree of independent adjustability for the size of the squark masses, such that they can be set not very far from their experimental bound of $\approx 300 \mathrm{GeV}$. The first feature allows us to evade the LEP II bound of $M_{\text {Higgs }} \gtrsim 114 \mathrm{GeV}$ without relying on large radiative corrections from top-stop loops, and thus to have small top squark masses. The second and third features then facilitate a softening of the top-stop loop correction to the up-type Higgs mass-squared parameter to a level commensurable with the Higgs field VEV $v \simeq 174 \mathrm{GeV}$, thereby eliminating the need for delicate cancellations. Specifically, the low mass-generation scale leads to a modest logarithm, $\simeq(2 \sim 5)$, in the correction, and the free adjustability of the squark masses moderates the overall mass scale of the correction.

In the first class of models, the additional Higgs quartic contribution is provided by $F$ exchange of a singlet chiral superfield. A fundamental intermediate-scale supersymmetry breaking is communicated to the MSSM fields primarily via two means. First, a new $U(1)$ gauge superfield acquires a $D$-term VEV at the intermediate scale and marries the 321 gauginos with adjoint chiral fermions via nonrenormalizable interactions. This leads to a consequence that the radiative generation of the sfermion masses occurs at a scale only a factor of $O(4 \pi)$ higher than the electroweak scale. This accomplishes the low scale of sfermion mass generation. The adjustability of squark masses is accomplished simply by making the nonrenormalizable couplings responsible for the gaugino masses independent for $S U(3)_{C}, S U(2)_{L}$, and $U(1)_{Y}$. The second means for transmitting supersymmetry breaking is via supergravity effects. These naturally generate weak-scale supersymmetric and supersymmetry breaking masses for the Higgs and singlet fields, producing an appropriate Higgs sector superpotential. Working together, these charac- 
teristic features of the models allow for the coexistence of the electroweak VEV with a variety of superparticle spectra, with a very mild tuning of about $20 \%$.

The second class of models also relies on $F$-exchange of a singlet to obtain the additional Higgs quartic coupling. However, communication of supersymmetry breaking to the MSSM sector more closely follows traditional gauge mediation. This leads to sfermion mass-generation scales $O\left(16 \pi^{2}\right)$ higher than the electroweak scale, which are larger than the mass-generation scales for the first class of models, but still provides only modest logarithmic enhancement in the Higgs mass correction. Supersymmetry is fundamentally broken at an intermediate scale, at a location physically separated from the MSSM fields in an extra dimension. The breaking is then communicated between the two locations by a bulk $U(1)$, which ultimately gives supersymmetric and supersymmetry breaking masses of $O(10 \sim 100 \mathrm{TeV})$ to the messenger fields of gauge mediation. We assume no specific relation between any of the mass parameters for the messengers, ensuring the adjustability of the squark masses. Weak-scale values for supersymmetric and supersymmetry breaking masses for the Higgs and singlet fields are again generated from supergravity effects. Within this class of models the reduction of tunings to $(10 \sim 20) \%$ can be obtained, again with a variety of configurations for the superparticle spectrum.

In searching out parameter points of reduced tuning in the above models, it quickly becomes apparent that the weakest tunings and largest Higgs boson masses are generally obtained when the Higgs potential is made stable along both the $H_{u}$ and $H_{d}$ axes, and destabilized in an intermediate direction via the $\mu B$ term. The reason is simply that the Higgs quartic coupling due to $F$-exchange is strongest when $\tan \beta \sim 1$. Thus, to most successfully utilize the new quartic coupling, smaller values for $\tan \beta$ are preferred, and electroweak symmetry breaking must be dominantly caused by the $\mu B$ term. This clearly demonstrates the framework introduced in [18]. It is very encouraging that this very generic idea has been successfully demonstrated in two distinct classes of realistic models.

\section{Acknowledgments}

This work was supported in part by the Director, Office of Science, Office of High Energy and Nuclear Physics, of the US Department of Energy under Contract DE-AC02-05CH11231. The work of Y.N. was also supported by the National Science Foundation under grant PHY0403380, by a DOE Outstanding Junior Investigator award, and by an Alfred P. Sloan Research Fellowship. 


\section{References}

[1] S. Dimopoulos and H. Georgi, Nucl. Phys. B 193, 150 (1981); N. Sakai, Z. Phys. C 11, 153 (1981); S. Dimopoulos, S. Raby and F. Wilczek, Phys. Rev. D 24, 1681 (1981).

[2] Z. Chacko, Y. Nomura and D. Tucker-Smith, Nucl. Phys. B 725, 207 (2005) [arXiv:hep$\mathrm{ph} / 0504095]$.

[3] Y. Nomura and B. Tweedie, Phys. Rev. D 72, 015006 (2005) [arXiv:hep-ph/0504246].

[4] J. R. Ellis, J. F. Gunion, H. E. Haber, L. Roszkowski and F. Zwirner, Phys. Rev. D 39, 844 (1989).

[5] M. Cvetic, D. A. Demir, J. R. Espinosa, L. L. Everett and P. Langacker, Phys. Rev. D 56, 2861 (1997) [Erratum-ibid. D 58, 119905 (1998)] [arXiv:hep-ph/9703317].

[6] J. R. Espinosa and M. Quiros, Phys. Rev. Lett. 81, 516 (1998) [arXiv:hep-ph/9804235].

[7] P. Batra, A. Delgado, D. E. Kaplan and T. M. P. Tait, JHEP 0402, 043 (2004) [arXiv:hepph/0309149]; A. Maloney, A. Pierce and J. G. Wacker, arXiv:hep-ph/0409127.

[8] J. A. Casas, J. R. Espinosa and I. Hidalgo, JHEP 0401, 008 (2004) [arXiv:hep-ph/0310137]; A. Brignole, J. A. Casas, J. R. Espinosa and I. Navarro, Nucl. Phys. B 666, 105 (2003) [arXiv:hep-ph/0301121].

[9] R. Harnik, G. D. Kribs, D. T. Larson and H. Murayama, Phys. Rev. D 70, 015002 (2004) [arXiv:hep-ph/0311349]; S. Chang, C. Kilic and R. Mahbubani, Phys. Rev. D 71, 015003 (2005) [arXiv:hep-ph/0405267]; A. Delgado and T. M. P. Tait, arXiv:hep-ph/0504224.

[10] A. Birkedal, Z. Chacko and Y. Nomura, Phys. Rev. D 71, 015006 (2005) [arXiv:hep$\mathrm{ph} / 0408329]$.

[11] K. S. Babu, I. Gogoladze and C. Kolda, arXiv:hep-ph/0410085.

[12] R. Kitano and Y. Nomura, arXiv:hep-ph/0509039.

[13] K. Choi, K. S. Jeong and K. i. Okumura, arXiv:hep-ph/0504037; K. Choi, K. S. Jeong, T. Kobayashi and K. i. Okumura, arXiv:hep-ph/0508029.

[14] Y. Nomura, D. Tucker-Smith and B. Tweedie, Phys. Rev. D 71, 075004 (2005) [arXiv:hep$\mathrm{ph} / 0403170]$.

[15] G. F. Giudice and A. Masiero, Phys. Lett. B 206, 480 (1988).

[16] P. J. Fox, A. E. Nelson and N. Weiner, JHEP 0208, 035 (2002) [arXiv:hep-ph/0206096].

[17] Y. Nomura and T. Yanagida, Phys. Lett. B 487, 140 (2000) [arXiv:hep-ph/0005211].

[18] Y. Nomura, D. Poland and B. Tweedie, arXiv:hep-ph/0509244. 
[19] M. Bastero-Gil, C. Hugonie, S. F. King, D. P. Roy and S. Vempati, Phys. Lett. B 489, 359 (2000) [arXiv:hep-ph/0006198]; R. Dermisek and J. F. Gunion, Phys. Rev. Lett. 95, 041801 (2005) [arXiv:hep-ph/0502105].

[20] T. Gregoire, R. Rattazzi and C. A. Scrucca, arXiv:hep-ph/0505126.

[21] L. Carpenter, P. J. Fox and D. E. Kaplan, arXiv:hep-ph/0503093.

[22] Z. Chacko, P. J. Fox and H. Murayama, Nucl. Phys. B 706, 53 (2005) [arXiv:hep$\mathrm{ph} / 0406142]$.

[23] L. Randall and R. Sundrum, Nucl. Phys. B 557, 79 (1999) [arXiv:hep-th/9810155].

[24] M. Masip, R. Munoz-Tapia and A. Pomarol, Phys. Rev. D 57, 5340 (1998) [arXiv:hep$\mathrm{ph} / 9801437]$.

[25] S. Eidelman et al. [Particle Data Group], Phys. Lett. B 592 (2004) 1.

[26] G. W. Anderson and D. J. Castano, Phys. Lett. B 347, 300 (1995) [arXiv:hep-ph/9409419]; see also R. Barbieri and G. F. Giudice, Nucl. Phys. B 306, 63 (1988).

[27] See, e.g., F. M. Borzumati and C. Greub, Phys. Rev. D 58, 074004 (1998) [arXiv:hepph/9802391]; Phys. Rev. D 59, 057501 (1999) [arXiv:hep-ph/9809438]; M. Ciuchini, G. Degrassi, P. Gambino and G. F. Giudice, Nucl. Phys. B 527, 21 (1998) [arXiv:hep-ph/9710335]; P. Gambino and M. Misiak, Nucl. Phys. B 611, 338 (2001) [arXiv:hep-ph/0104034]; M. Neubert, Eur. Phys. J. C 40, 165 (2005) [arXiv:hep-ph/0408179].

[28] M. Dine and W. Fischler, Phys. Lett. B 110, 227 (1982); Nucl. Phys. B 204, 346 (1982); L. Alvarez-Gaume, M. Claudson and M. B. Wise, Nucl. Phys. B 207, 96 (1982); S. Dimopoulos and S. Raby, Nucl. Phys. B 219, 479 (1983).

[29] M. Dine, A. E. Nelson and Y. Shirman, Phys. Rev. D 51, 1362 (1995) [arXiv:hepph/9408384]; M. Dine, A. E. Nelson, Y. Nir and Y. Shirman, Phys. Rev. D 53, 2658 (1996) [arXiv:hep-ph/9507378].

[30] K. Agashe and M. Graesser, Nucl. Phys. B 507, 3 (1997) [arXiv:hep-ph/9704206]; S. P. Martin, Phys. Rev. D 55, 3177 (1997) [arXiv:hep-ph/9608224].

[31] G. F. Giudice, M. A. Luty, H. Murayama and R. Rattazzi, JHEP 9812, 027 (1998) [arXiv:hep-ph/9810442].

[32] Y. Kawamura, Prog. Theor. Phys. 105, 999 (2001) [arXiv:hep-ph/0012125]; L. J. Hall and Y. Nomura, Phys. Rev. D 64, 055003 (2001) [arXiv:hep-ph/0103125]; Annals Phys. 306, 132 (2003) [arXiv:hep-ph/0212134].

[33] L. Randall and R. Sundrum, Phys. Rev. Lett. 83, 3370 (1999) [arXiv:hep-ph/9905221]. 
[34] T. Gherghetta and A. Pomarol, Nucl. Phys. B 586, 141 (2000) [arXiv:hep-ph/0003129].

[35] W. D. Goldberger, Y. Nomura and D. R. Smith, Phys. Rev. D 67, 075021 (2003) [arXiv:hep$\mathrm{ph} / 0209158]$.

[36] J. M. Maldacena, Adv. Theor. Math. Phys. 2, 231 (1998) [Int. J. Theor. Phys. 38, 1113 (1999)] [arXiv:hep-th/9711200].

[37] N. Arkani-Hamed, M. Porrati and L. Randall, JHEP 0108, 017 (2001) [arXiv:hepth/0012148]; R. Rattazzi and A. Zaffaroni, JHEP 0104, 021 (2001) [arXiv:hep-th/0012248].

[38] M. A. Luty and R. Sundrum, Phys. Rev. D 65, 066004 (2002) [arXiv:hep-th/0105137].

[39] Y. Nomura and K. Suzuki, Phys. Rev. D 68, 075005 (2003) [arXiv:hep-ph/0110040]. 